\title{
False aneurysm formation after Salmonella virchow infection of a pre-existent ventricular aneurysm - survival after surgical resection
}

\author{
S. Echevarria, R. Arjona, J. Alonso, J.A. Riancho, J.M. Revuelta and J. Gonzalez \\ Macias
}

Departments of Internal Medicine and Cardiovascular Surgery, National Hospital 'Marques de Valdecilla', Faculty of Medicine, University of Santander, Santander, Spain.

\begin{abstract}
Summary: A 55 year old man with a left ventricular aneurysm, secondary to a previous myocardial infarction, was hospitalized due to a Salmonella virchow bacteraemia. During a 3-week hospital course he was persistently bacteraemic and febrile despite antibiotic treatment. Gallium isotope scanning and 2-D-echocardiography were helpful in demonstrating the presence of an infected false aneurysm at the site of a true aneurysm. Surgical resection in addition to prolonged antibiotic therapy was necessary for cure.
\end{abstract}

\section{Introduction}

Despite improvement in sanitation and careful monitoring of food processing, salmonella infections are among the most prevalent communicable bacterial diseases in the world. ${ }^{1}$ While salmonellosis is often considered to affect primarily the gastrointestinal tract, infection at other sites may occur. ${ }^{2}$

Endothelial infections are rare complications of salmonellosis, as these organisms appear to have an avidity for abnormal endothelial surfaces. ${ }^{3}$ Diagnosis of such infections is often difficult and too frequently not established until the patient has advanced disease or dies and post-mortem examination is done. Endovascular infection involving an atherosclerotic abdominal aortic aneurysm is the most reported endothelial manifestation of salmonellosis. ${ }^{4,5}$ In addition, cardiac complications including endocarditis, pericarditis, myocarditis and coronary arteritis have been reported. ${ }^{2}$

We describe a case of left ventricular pseudoaneurysm due to Salmonella virchow infection, in which gallium scanning and echocardiography allowed a diagnosis to be made before a fatal cardiac rupture occurred.

\section{Case report}

A 55 year old man was admitted to the hospital because of general malaise and fever. Past medical

Correspondence: S. Echevarria, M.D., Avda. Cardenal Herrera Oria, 31-C, $5^{\circ} \mathrm{D}, 39011$ Santander, Spain

Accepted: 17 August 1988 history included an anterolateral myocardial infarc-

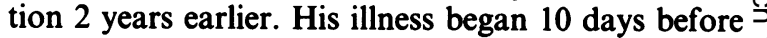
admission, with intermittent fever up to $39^{\circ} \mathrm{C}$, chills, $\vec{\otimes}$ dry cough and general malaise. He denied chest pain, dyspnoea, abdominal pain, diarrhoea and other symos. toms.

He was acutely ill, with temperature $39^{\circ} \mathrm{C}$, heart rate $88 / \mathrm{min}$, and blood pressure of $100 / 60 \mathrm{mmHg}$. There was a broadened apex beat, but no cardiac murmurs, $\stackrel{\perp}{\perp}$ rub or gallop. Abdominal examination showed $\overrightarrow{\vec{F}}$ splenomegaly $2 \mathrm{~cm}$ below the left costal margin.

Laboratory data included haemoglobin $12 \mathrm{~g} / \mathrm{dl}$, white blood cell count $5.4 \times 10^{6} / 1$ and sedimentation rate $29 \mathrm{~mm} / \mathrm{h}$. Routine biochemistry and urinalysis were normal. The chest $\mathrm{X}$-ray showed cardiomegaly with left ventricular prominence and clear lung fields. The electrocardiogram showed sinus rhythm, rate $88 / \mathrm{min}$, and evidence of old myocardial infarct. Cultures of six blood samples obtained on the first hospital day contained Gram-negative rods identified as Salmonella virchow sensitive to chloramphenicol, $\frac{7}{0}$ amoxycillin and trimethoprim-sulphamethoxazole. Sputum, urine and stool cultures were negative. A first $\sigma$ two-dimensional echocardiogram showed an apical N left ventricular aneurysm. No valvular vegetations N could be visualized. Treatment with chloramphenicol was begun.

Despite antibiotic therapy, the patient continued to have fevers up to $39.5^{\circ} \mathrm{C}$, and $S$. virchow grew again in successive blood cultures taken on the following days. On the 10th hospital day, chloramphenicol was dis- 0 continued and amoxycillin was begun. A computed $\stackrel{\square}{\square}$ tomographic scan of the abdomen was normal. $\stackrel{\mathbb{Q}}{\Omega}$ 
Gallium-67 citrate scanning showed uptake over the apex of the heart. A repeated two-dimensional echocardiogram demonstrated the existence of a false aneurysm arising from the previous ventricular aneurysm and containing thrombotic material.

On the 20th hospital day urgent operation was undertaken. At surgery, a left ventricular aneurysm with a large mural thrombus was present. A false aneurysm with a diameter of $3 \mathrm{~cm}$ originating from the ventricular aneurysm and containing laminated thrombus was found. Aneurysmectomy was successfully completed. On microscopic examination, the wall of the false aneurysm was seen to consist of dense fibrous tissue with extensive inflammatory response and microabscesses. Cultures of the tissue grew S. virchow.

Postoperatively defervescence was prompt and the patient remained afebrile throughout the subsequent 3 weeks of oral antibiotic therapy with amoxycillin. After discharge the patient has remained afebrile and in good health. Repeated blood cultures have been sterile.

\section{Discussion}

The development of a ventricular false aneurysm requires a fibrous thickening and adhesions of the pericardium and epicardium sufficient to localize and retain the escaping blood after a myocardial rupture within a restricted area. Contained by the pericardium, its wall is composed of organized haematoma lacking any elements of the myocardial wall. Although the exact incidence is difficult to determine, a false aneurysm develops in a small proportion of patients with cardiac rupture. ${ }^{6,7}$ Most reported cases have been caused by myocardial infarction or cardiac surgery, and among the less frequent causes are endocarditis, myocardial abscess and trauma. ${ }^{8-10}$

The distinctive radiological, angiographic and pathological features of false aneurysms of the left ventricle have been previously described. ${ }^{6,7,11-13}$ Clinically, the false aneurysm differs little from the true aneurysm in its tendency to produce heart failure, systemic embolization or arrhythmia ${ }^{6,9}$ However, the incidence of fatal cardiac rupture is much higher in false aneurysms. ${ }^{11,14,15}$ This risk of rupture and the potential for successful surgical resection ${ }^{10}$ emphasize the need to recognize this complication.

In the patient described here, a ventricular aneurysm resulting from a previous myocardial infarction represented a nidus for bacterial growth during salmonella bacteraemia. We believe that the superimposed bacterial infection contributed to the formation of the false aneurysm, and that this case was the end result of a train of events which from an initial salmonella infection led to bacteraemia, mural endocarditis and subsequently to direct spread into the underlying tissues.

Non-typhoidal salmonella seems to have a particular tendency to colonize abnormal endothelial surfaces. ${ }^{3}$ Infection of an atherosclerotic abdominal aortic aneurysm is among the most reported endothelial manifestation of salmonellosis. ${ }^{2,4,5}$ Although the heart is infrequently involved, cardiac complications, especially mural endocarditis, ${ }^{2}$ have been reported. Other cardiac manifestations described included myocarditis, ${ }^{2}$ pericarditis, coronary arteritis and myocardial abscesses. ${ }^{2,16-18}$ The usual portal of entry for these organisms is the gastrointestinal tract, and abnormal intracardiac endothelium represented a nidus for bacterial growth.

Infections involving a ventricular aneurysm represent a small subset of intracardiac infections. Gramnegative bacilli appear to be the most common pathogens, ${ }^{19}$ and when these intracardiac infections do occur, antemortem diagnosis has been difficult resulting in significant mortality. A few cases of infected true or false aneurysms due to salmonella have been reported $^{7,20-23}$ although these organisms are the principal causative agents of infected aneurysms. In our patient, gallium isotope scanning and 2Dechocardiography were valuable in diagnosis; these techniques may assist in earlier detection in future cases.

Medical therapy alone was not effective in our patient. Extensive surgical resection and prolonged antibiotic treatment was necessary for cure, and to our knowledge this is the first reported description of a patient with a false aneurysm infected by salmonella who survived. This parallels the experience of others who have found that once a salmonella spp. has infected the heart or major blood vessels, it has been extremely difficult to eradicate with antibiotics alone. . $^{2,3,24}$

We believe that persons older than age 50 with salmonella bacteraemia, particularly if they have previous cardiovascular lesions, appear to constitute a high-risk group for endothelial infection. Therefore, although we agree with the recommendation against routine antibiotic treatment for salmonella gastroenteritis, antibiotic therapy should be considered in high-risk patients to prevent the development of an endothelial focus. 


\section{References}

1. Centers for Disease Control. Human Salmonella isolates. United States 1983. MMWR 1984, 33: 693-695.

2. Cohen, J.I., Bartlett, J.A. \& Corey, G.R. Extra-intestinal manifestations of Salmonella infections. Medicine (Baltimore) 1987, 66: 349-388.

3. Cohen, P.S., O'Brien, T.F., Schoenbaum, S.C. \& Medeiros, A.A. The risk of endothelial infection in adults with Salmonella bacteremia. Ann Intern Med 1978, 89: 931-932.

4. Kanwar, Y.S., Malhotra, V., Andersen, B.R. \& Pilz, C.G. Salmonella associated with abdominal aortic aneurysm. Arch Intern Med 1974, 134: 1095-1098.

5. Parsons, R., Gregory, J. \& Palmer, D.L. Salmonella infections of the abdominal aorta. Rev Infect Dis 1983, 5: 227-231.

6. Sweet, S.E., Sterling, R., McCormick, J.R., Klein, M.D., Berger, R.L. \& Ryan, T.J. Left ventricular false aneurysm after coronary bypass surgery: Radionuclide diagnosis and surgical resection. Am J Cardiol 1979, 43: $154-157$.

7. Catheerwood, E., Mintz, G.S., Kotler, M.N., Kimbiris, D., Lemmon, W. \& Parry, W.R. Pseudoaneurysm of the left ventricle complicated by Salmonella typhimurium infection. Recognition by two-dimensional echocardiography. Am J Med 1980, 68: 782-786.

8. Davidson, K.H., Parisi, A.F., Harrington, J.J., Barsamian, E.M. \& Fishbein, M.C. Pseudoaneurysm of the left ventricle: an unusual echocardiographic presentation. Review of the literature. Ann Intern Med 1977, 86: 430-433.

9. Martin, R.H., Almond, C.H., Saab, S. \& Watson, L.E. True and false aneurysms of the left ventricle following myocardial infarction. Am J Med 1977, 62: 418-424.

10. Yakierevitch, V., Vidne, B., Melamed, R. \& Levy, M.J. False aneurysm of the ventricle. Surgical treatment. $J$ Thorac Cardiovasc Surg 1978, 76: 556-558.

11. Higgins, C.B., Lipton, M.J., Johnson, A.D., Peterson, K.L. \& Vieweg, W.V.R. False aneurysms of the left ventricle. Identification of distinctive clinical, radiographic and angiographic features. Radiology 1987, 127: 21-27.

12. Sears, T.D., Ong, Y.S., Starke, H. \& Forker, A.D. Left ventricular pseudoaneurysm identified by cross-sectional echocardiography. Ann Int Med 1979, 90: 935-936.
13. Botvinick, E.H., Shames, D., Hutchinson, J.C., Roé, B.B. \& Fitzpatrick, M. Noninvasive diagnosis of a false left ventricular aneurysm with radioisotope injected cardiac blood pool imaging. Differentiation from the aneurysm. Am J Cardiol 1976, 37: 1089-1093.

14. Vlodaver, Z., Coe, J.L. \& Edwards, J.E. True and false left ventricular aneurysms: propensity for the latter to rupture. Circulation 1975, 51: 567-572.

15. Van Tassel, R.A. \& Edwards, J.E. Rupture of heart complicating myocardial infarction. Analysis of $\mathbf{4 0}$ cases including nine examples of left ventricular false aneurysm. Chest 1972, 61: 104-116.

16. Sanders, V., Misanik, L.F. Salmonella myocarditis. Report of a case with ventricular rupture. Am Heart $J$ 1964, 68: 682-685.

17. Doraiswami, S., Friedmen, S.A., Kagan, A. \& Gladstone, J.L. Salmonella endocarditis complicated by a myocardial abscess. Am J Cardiol 1970, 26: 102-105.

18. Langaker, O.M. \& Suanes, K. Myocardial abscess due to Salmonella typhimurium. Br Heart J 1973, 35: 871-873. .

19. Venezio, F.R., Thompson, J.E., Sullivan, H., Subramanian, R., Ritzman, P. \& Gunnar, R.M. Infection of a ventricular aneurysm and cardiac mural thrombus. Survival after surgical resection. Am J Med 1984, 77: $551-554$.

20. Decker, J.P. \& Clancy, C.F. Salmonella endocarditis Report of three cases including one of mural endocard itis. Bull Ayer Clinic Lab 1959, 4: 1-8.

21. McNally, E.M., Kennedy, R.J., Grace, W.R. Salmonel通 infantis infection of a pre-existent ventricular aneurysmo Am Heart J 1964, 68: 541-548.

22. Connely, G.P., Matthay, R.A., Sponzo, R.W. \& Smith F.E. Salmonella typhimurium abscess formation in a calcified ventricular aneurysm. Chest 1974, 66: 457-459.

23. Kortleve, J.W., Duren, D.R. \& Becker, A.E. Cardiac aneurysm complicated by Salmonella abscess. A clinicopathological correlation in two patients. $A m ~ J$ Med 1980, 68: 395-340.

24. Aguado, J.M., Fernandez-Guerrero, M.L., La Banda, F. \& Garces, J.L.G. Salmonella infections of the abdominal aneurysm cured with prolonged antibiotic treatment. $J$ Infect 1978, 14: 135-139. 